\title{
Diversion of Controlled Drugs in Hospitals: A Scoping Review of Contributors and Safeguards
}

\author{
Mark Fan, BASc, MHSc ${ }^{1 *}$; Dorothy Tscheng, RPh, BScPhm, CGP2; Michael Hamilton, BSc, BEd, MD, MPH, CCFP2; \\ Bridgett Hyland, PharmD Candidate'; Rachel Reding, BASc, MHSc'; Patricia Trbovich, PhD ${ }^{1,3}$
}

${ }^{1}$ HumanEra, Office of Research and Innovation, North York General Hospital, Toronto, Ontario, Canada; ${ }^{2}$ Institute for Safe Medication Practices Canada, Toronto, Ontario, Canada; Institute of Health Policy, Management and Evaluation, University of Toronto, Toronto, Ontario, Canada.

Drug losses and theft from the healthcare system are accelerating; hospitals are pressured to implement safeguards to prevent drug diversion. Thus far, no reviews summarize all known risks and potential safeguards for hospital diversion. Past incidents of hospital drug diversion have impacted patient and staff safety, increased hospital costs, and resulted in infectious disease outbreaks. We searched MEDLINE, Embase, PsycINFO, CINAHL, Scopus, and Web of Science databases and the gray literature for articles published between January 2005 and June 2018. Articles were included if they focused on hospital settings and discussed either: (1) drug security or accounting practices (any drug) or (2) medication errors, healthcare worker substance use disorder, or incident reports (only with reference to controlled drugs). We included 312 articles and extracted four categories of data: (1) article characteristics (eg, author location), (2) article focus (eg, clinical areas discussed), (3) contributors to diversion (eg, factors enabling drug theft), and (4) diversion safeguards. Literature reveals a large number of contributors to drug diversion in all stages of the medication-use process. All health professions and clinical units are at risk. This review provides insights into known methods of diversion and the safeguards hospitals must consider to prevent them. Careful configuration of healthcare technologies and processes in the hospital environment can reduce the opportunity for diversion. These system-based strategies broaden the response to diversion beyond that of individual accountability. Further evidence is urgently needed to address the vulnerabilities outlined in this review and prevent harm. Journal of Hospital Medicine 2019;14:419-428. Published online first June 12, 2019. () 2019 Society of Hospital Medicine

\section{T} he United States (US) and Canada are the two highest per-capita consumers of opioids in the world; ${ }^{1}$ both are struggling with unprecedented opioid-related mortality. ${ }^{2,3}$ The nonmedical use of opioids is facilitated by diversion and defined as the transfer of drugs from lawful to unlawful channels of use $\mathrm{e}^{4,5}$ (eg, sharing legitimate prescriptions with family and friends $\left.{ }^{6}\right)$. Opioids and other controlled drugs are also diverted from healthcare facilities; ${ }^{4,5,7,8}$ Canadian data suggest these incidents may be increasing (controlled-drug loss reports have doubled each year since $2015^{9}$ ).

The diversion of controlled drugs from hospitals affects patients, healthcare workers (HCWs), hospitals, and the public. Patients suffer insufficient analgesia or anesthesia, experience substandard care from impaired HCWs, and are at risk of infections from compromised syringes. ${ }^{4,10,11} \mathrm{HCW}$ s that divert are at risk of overdose and death; they also face regulatory censure, criminal prosecution, and civil malpractice suits. ${ }^{12,13}$ Hospitals bear the cost of diverted drugs, ${ }^{14,15}$ internal investigations, ${ }^{4}$ and follow-up care for affected patients, ${ }^{4,13}$ and can be fined in

*Corresponding Author: Mark Fan, E-mail: mark.fan@nygh.on.ca; Telephone: 416-756-6000 ×3075

Additional Supporting Information may be found in the online version of this article.

Received: December 11, 2018; Revised: April 15, 2019;

Accepted: April 23, 2019

๑) 2019 Society of Hospital Medicine DOI 10.12788/jhm.3228 excess of $\$ 4$ million dollars for inadequate safeguards. ${ }^{16} \mathrm{Neg}$ ative publicity highlights hospitals failing to self-regulate and report when diversion occurs, compromising public trust. . $^{17-19}$ Finally, diverted drugs impact population health by contributing to drug misuse.

Hospitals face a critical problem: how does a hospital prevent the diversion of controlled drugs? Hospitals have not yet implemented safeguards needed to detect or understand how diversion occurs. For example, $79 \%$ of Canadian hospital controlled-drug loss reports are "unexplained losses," demonstrating a lack of traceability needed to understand the root causes of the loss. A single US endoscopy clinic showed that $\$ 10,000$ of propofol was unaccounted for over a four-week period. ${ }^{14}$ Although transactional discrepancies do not equate to diversion, they are a potential signal of diversion and highlight areas for improvement. ${ }^{15}$ The hospital medication-use process (MUP; eg, procurement, storage, preparation, prescription, dispensing, administration, waste, return, and removal) has multiple vulnerabilities that have been exploited. Published accounts of diversion include falsification of clinical documents, substitution of saline for medication, and theft., ${ }^{40-23}$ Hospitals require guidance to assess their drug processes against known vulnerabilities and identify safeguards that may improve their capacity to prevent or detect diversion.

In this work, we provide a scoping review on the emerging topic of drug diversion to support hospitals. Scoping reviews can be a "preliminary attempt to provide an overview of exist- 
ing literature that identifies areas where more research might be required. "24 Past literature has identified sources of drugs for nonmedical use, $6,25,26$ provided partial data on the quantities of stolen drug, ${ }^{7,8}$ and estimated the rate of HCW diversion. ${ }^{5,27-29}$ However, no reviews have focused on system gaps specific to hospital MUPs and diversion. Our review remedies this knowledge gap by consolidating known weaknesses and safeguards from peer- and nonpeer-reviewed articles. Drug diversion has been discussed at conferences and in news articles, case studies, and legal reports; excluding such discussion ignores substantive work that informs diversion practices in hospitals. Early indications suggest that hospitals have not yet implemented safeguards to properly identify when diversion has occurred, and consequently, lack the evidence to contribute to peer-reviewed literature. This article summarizes (1) clinical units, health professions, and stages of the MUP discussed, (2) contributors to diversion in hospitals, and (3) safeguards to prevent or detect diversion in hospitals.

\section{METHODS}

\section{Scoping Review}

We followed Arksey and O'Malley's six-step framework for scoping reviews, ${ }^{30}$ with the exception of the optional consultation phase (step 6). We addressed three questions (step 1): what clinical units, health professions, or stages of the medication-use process are commonly discussed; what are the identified contributors to diversion in hospitals; and what safeguards have been described for prevention or detection of diversion in hospitals? We then identified relevant studies (step 2) by searching records published from January 2005 to June 2018 in MEDLINE, Embase, PsycINFO, CINAHL, Scopus, and Web of Science; the gray literature was also searched (see supplementary material for search terms).

All study designs were considered, including quantitative and qualitative methods, such as experiments, chart reviews and audit reports, surveys, focus groups, outbreak investigations, and literature reviews. Records were included (step 3) if abstracts met the Boolean logic criteria outlined in Appendix 1. If no abstract was available, then the full-text article was assessed. Prior to abstract screening, four reviewers (including R.R.) independently screened batches of 50 abstracts at a time to iteratively assess interrater reliability (IRR). Disagreements were resolved by consensus and the eligibility criteria were refined until IRR was achieved (Fleiss kappa > 0.65). Once IRR was achieved, the reviewers applied the criteria independently. For each eligible abstract, the full text was retrieved and assigned to a reviewer for independent assessment of eligibility. The abstract was reviewed if the full-text article was not available. Only articles published in English were included.

Reviewers charted findings from the full-text records (steps 4 and 5) by using themes defined a priori, specifically literature characteristics (eg, authors, year of publication), characteristics related to study method (eg, article type), variables related to our research questions (eg, variations by clinical unit, health profession), contributors to diversion, and safeguards to detect or prevent diversion. Inductive additions or modifications to the themes were proposed during the full-text review (eg, reviewers added a theme "name of drugs diverted" to identify drugs frequently reported as diverted) and accepted by consensus among the reviewers.

\section{RESULTS}

Scoping Review

The literature search generated 4,733 records of which 307 were duplicates and 4,009 were excluded on the basis of the eligibility criteria. The reviewers achieved $100 \%$ interrater agreement on the fourth round of abstract screening. Upon full-text review, 312 articles were included for data abstraction (Figure).

\section{Literature Characteristics}

Table 1 summarizes the characteristics of the included literature. The articles were published in a mix of peer-reviewed (137, 44\%) and nonpeer-reviewed (175, 56\%) sources. Some peer-reviewed articles did not use research methods, and some nonpeer-reviewed articles used research methods (eg, doctoral theses). Therefore, Table 1 categorizes the articles by research method (if applicable) and by peer-review status. The articles primarily originated in the United States $(211,68 \%)$ followed by Canada (79, 25\%) and other countries (22,7\%). Most articles were commentaries, editorials, reports or news media, rather than formal studies presenting original data.

\section{Literature Focus by Clinical Unit, Health Profession, and Stage of Medication-Use Process}

Most articles did not focus the discussion on any one clinical unit, health profession, or stage of the MUP. Of the articles that made explicit mention of clinical units, hospital pharmacies and operating rooms were discussed most often, nurses were the most frequently highlighted health profession, and most stages of the MUP were discussed equally, with the exception of prescribing which was mentioned the least (Supplementary Table).

\section{Contributors to Diversion}

The literature describes a variety of contributors to drug diversion. Table 2 organizes these contributors by stage of the MUP and provides references for further discussion.

The diverse and system-wide contributors to diversion described in Table 2 support inappropriate access to controlled drugs and can delay the detection of diversion after it occurred. These contributors are more likely to occur in organizations that fail to adhere to drug-handling practices or to carefully review practices. ${ }^{34,44}$

\section{Diversion Safeguards in Hospitals}

Table 3 summarizes published recommendations to mitigate the risk of diversion by stage of the MUP.

\section{DISCUSSION}

This review synthesizes a broad sample of peer- and nonpeer-reviewed literature to produce a consolidated list of 


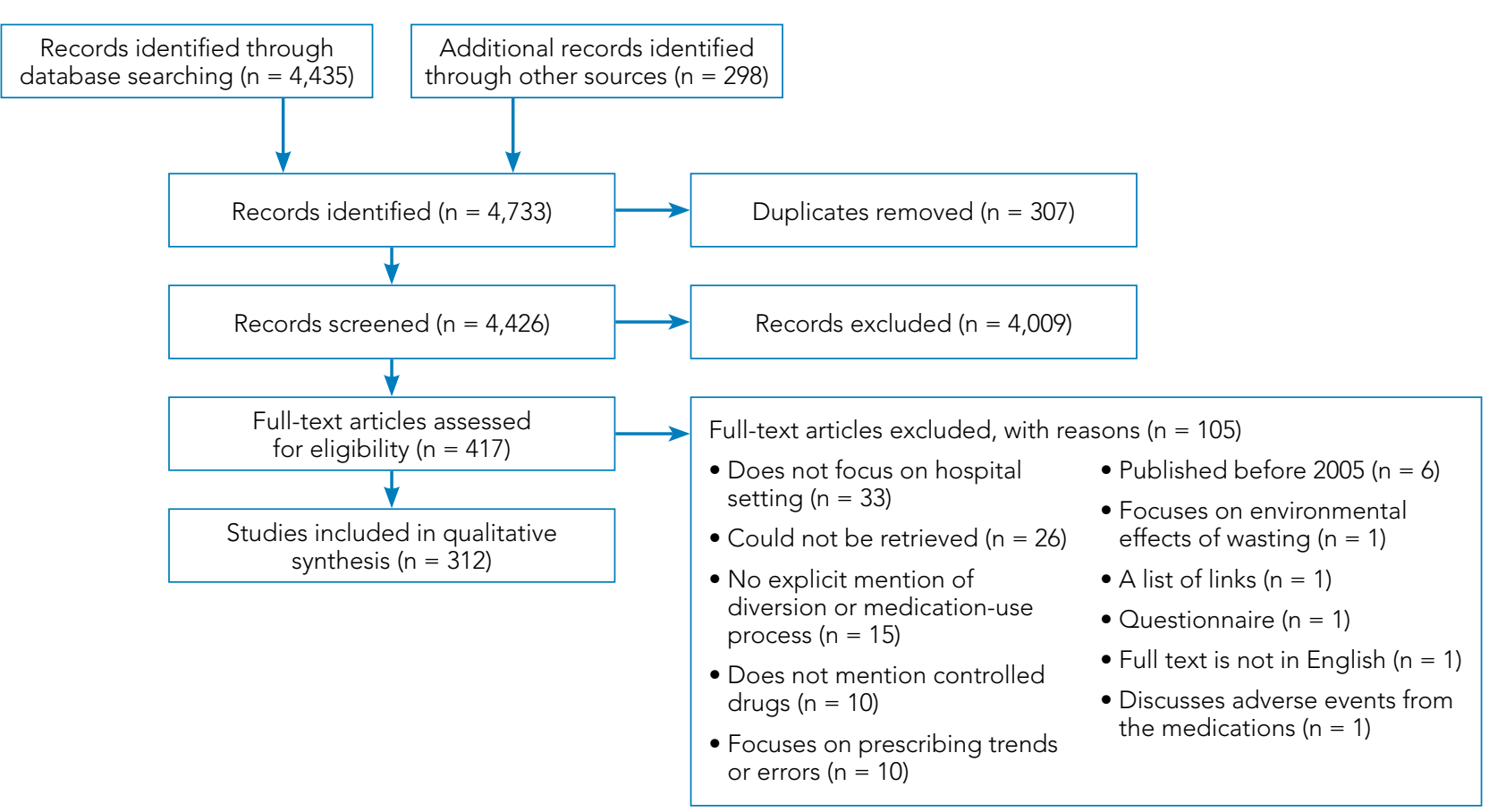

FIG. Flow Diagram of Inclusion and Exclusion of Identified Articles.

known contributors (Table 2) and safeguards against (Table 3) controlled-drug diversion in hospitals. The literature describes an extensive list of ways drugs have been diverted in all stages of the MUP and can be exploited by all health professions in any clinical unit. Hospitals should be aware that nonclinical $\mathrm{HCW}$ s may also be at risk (eg, shipping and receiving personnel may handle drug shipments or returns, housekeeping may encounter partially filled vials in patient rooms). Patients and their families may also use some of the methods described in Table 2 (eg, acquiring fentanyl patches from unsecured waste receptacles and tampering with unsecured intravenous infusions).

Given the established presence of drug diversion in the literature, ${ }^{5,7-9,96,97}$ hospitals should assess their clinical practices against these findings, review the associated references, and refer to existing guidance to better understand the intricacies of the topic. ${ }^{7,31,51,53,60,79}$ To accommodate variability in practice between hospitals, we suggest considering two underlying issues that recur in Tables 2 and 3 that will allow hospitals to systematically analyze their unique practices for each stage of the MUP.

The first issue is falsification of clinical or inventory documentation. Falsified documents give the opportunity and appearance of legitimate drug transactions, obscure drug diversion, or create opportunities to collect additional drugs. Clinical documentation can be falsified actively (eg, deliberately falsifying verbal orders, falsifying drug amounts administered or wasted, and artificially increasing patients' pain scores) or passively (eg, profiled automated dispensing cabinets [ADC] allow drug withdrawals for a patient that has been discharged or transferred over 72 hours ago because the system has not yet been updated). Falsification of inventory documentation can involve deliberate miscounting of drug inventory, removing records of drug procurement and intercepting the shipment when it arrives, and forging signatures on drug deliveries from the pharmacy to the care unit. Prevention safeguards include constraining clinical choices, decreasing delays to documentation updates, increasing traceability, and improving verification of transactions. For example, standardizing ordering protocols constrains clinical choices so that minimal controlled drug is dispensed, leading to reduced risk of dispensing more than the patient needs (eg, order sets that avoid dose ranges or limit as needed [PRN] doses). An example of decreasing delays to documentation updates are ADC profiles that rapidly remove discharged patients, so that it is not possible to dispense drugs for a transferred patient. Examples of increasing traceability include biometric (eg, fingerprint) signatures or using cameras in select areas which deter forgery and support auditing. Verification of the transactions listed in the documentation has typically relied upon a real-time witness, but may not always be possible. For example, it is infeasible to require a witness to verify all drug administration to patients. Therefore, future work may be needed to develop other strategies to verify physical transactions (eg, weight sensors and computer vision). Detection safeguards for documentation rely on auditing, therefore electronic systems can be an important asset to employ. For example, electronic systems support monitoring of unusual trends (eg, prescribing activity by care unit or $\mathrm{HCW}$; number of unverified verbal orders; dispensing activity by ADC, care unit, drug, or $\mathrm{HCW}$; variations in patient pain scores between $\mathrm{HCW}$; drug 
TABLE 1. Summary of Literature Characteristics ${ }^{1}$ ( $n=312$ Articles Included in the Analysis)

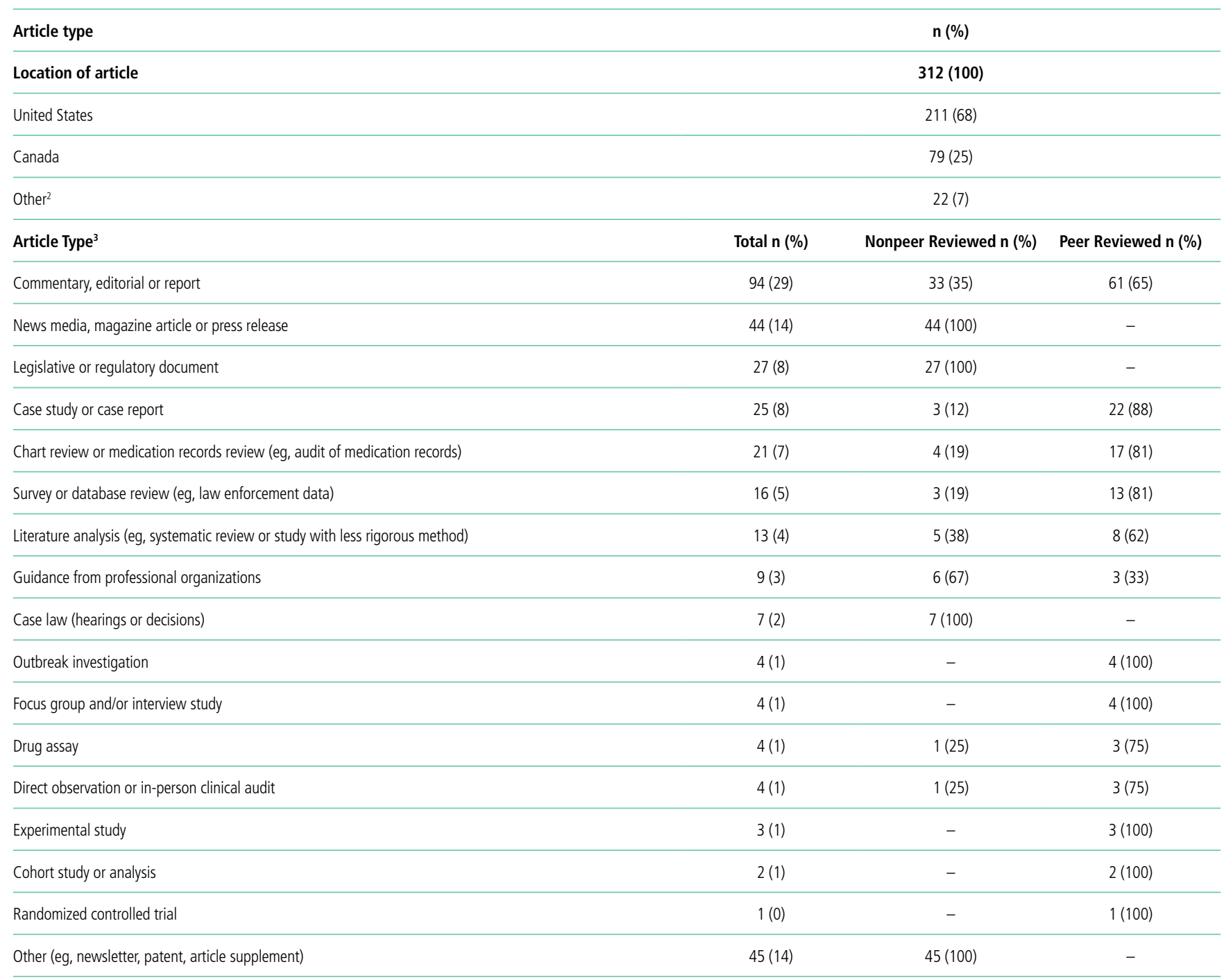

'All categories are listed by descending frequency (highest to lowest count), with the exception of the "Other" category.

Including Australia, Brazil, Finland, France, Japan, Korea, Malaysia, Nepal, New Zealand, South Africa, Spain, Thailand, United Kingdom

${ }^{3}$ Some articles were assigned to more than one category, therefore the total count of articles will exceed 312 .

wastage amounts). If data from multiple systems can be integrated (eg, electronic health records and ADCs), then hospitals can more easily identify discrepancies among the drug amount ordered, dispensed, administered, and wasted or disposed for each patient. Hospitals can also compare purchased inventory against financial records to identify discrepancies. Clinical outcomes can also highlight potential drug discrepancies (eg, uncontrolled pain could be a signal for partial or absent administration of drugs).

The second issue involves failure to maintain the physical security of controlled drugs, thereby allowing unauthorized access. This issue includes failing to physically secure drug stock (eg, propping doors open to controlled-drug areas; failing to log out of ADCs, thereby facilitating unauthorized access; and leaving prepared drugs unsupervised in patient care areas) or failing to maintain accurate access credentials (eg, staff no lon- ger working on the care unit still have access to the ADC or other secure areas). Prevention safeguards require adherence to existing security protocols (eg, locked doors and staff access frequently updated) and limiting the amount of controlled drugs that can be accessed (eg, supply on care unit should be minimized to what is needed and purchase smallest unit doses to minimize excess drug available to HCWs). Hospitals may need to consider if security measures are actually feasible for $\mathrm{HCW}$. For example, syringes of prepared drugs should not be left unsupervised to prevent risk of substitution or tampering; however, if the responsible HCW is also expected to collect supplies from outside the care area, they cannot be expected to maintain constant supervision. Detection safeguards include the use of tamper-evident packaging to support detection of compromised controlled drugs or assaying drug waste or other suspicious drug containers to detect dilution or tampering. 
TABLE 2. Contributors to Diversion at Each Stage of the Medication-Use Process with Associated List of References

\begin{tabular}{lll}
\hline Stage of Medication-Use Process & Contributors to Diversion & Description, Examples, and Associated References \\
\hline Procurement & Excess ordering & $\begin{array}{l}\text { Excess drug can be ordered and diverted by removing the purchase order and packing slip, thereby obscuring } \\
\text { evidence of any diversion at all. }{ }^{20,31}\end{array}$ \\
\hline Storage $^{a}$ & Unsupervised access to drug storage areas & $\begin{array}{l}\text { High levels of personnel traffic can lead to poor practices (eg, doors may be propped open }{ }^{32,33} \text { ), which may } \\
\text { obscure who has accessed drug storage areas. }{ }^{20,34}\end{array}$ \\
& $\begin{array}{l}\text { When unsupervised, drugs can be vulnerable to tampering, while the product container appears intact (eg, } \\
\text { removing a few pills from a 1000-count bottle, drinking cough syrup directly from the bottle } \\
\text { may also substitute saline for diverted drug to obscure tampering. }{ }^{21,22,31,36} \text { Intentional miscounts of received } \\
\text { drug while restocking can also obscure diversion. }{ }^{37,38}\end{array}$
\end{tabular}

\begin{tabular}{ll}
\hline Prescribing & Unverified verbal orders \\
\cline { 2 - 2 } & Flexible ordering
\end{tabular}
Verbal orders can be falsified to grant inappropriate access to controlled drug. ${ }^{12,31}$

Although there are legitimate reasons for allowing flexible dose orders (eg, as-needed doses, a dosing range), they allow access to more drug than may be needed and can facilitate diversion ${ }^{39,40}$ (eg, maximum doses recorded as administered, but excess actually diverted).

Forgery If prescription pads are not kept physically secured and strictly supervised, written orders can be altered, forged or reused. $23,31,36$

Preparation Compounding and repackaging

Procuring drugs that require compounding or repackaging (eg, not purchasing unit doses) provides opportunities for diversion (eg, diversion from overfill, ${ }_{15,31}$ "extra" withdrawals from multidose containers, tampering/substitution of drug in solutions). . $^{31,41}$

Dispensing Typical doses smaller than stocked drugs

When drug doses are purchased in formats that exceed the typical doses used on the clinical unit, and are not compounded or repackaged to unit doses, HCWs at the bedside gain reliable access to excess drug when prescribed. ${ }^{42}$

Poor verification of dispensing to clinical units When drugs are transferred from pharmacy to a clinical unit, the delivery person can forge the co-signature of an individual "verifying" receipt. ${ }^{35}$ Unsupervised inventory checks when replenishing unit inventory can open the door to intentional miscounts. ${ }^{37,43}$

Reduced pharmacy oversight of dispensing Implementation of $A D C s^{44}$ and computerized physician order entry can reduce awareness of drug use (eg, with introduction of technology hydromorphone previously available only from pharmacy may become available in the emergency department's $\left.A D C^{45}\right)$.

Dispensing and administration Loopholes in the intended use of ADCs

HCWs may cancel or perform null transactions, such that the ADC does not record a change in inventory, despite a quantity of drug having been taken, ${ }^{38,46-48}$ or they may withdraw both injectable and oral drugs at the same time (eg, a duplicate dose) to obscure diversion of an extra dose. ${ }^{34} \mathrm{HCW}$ s may withdraw drugs for patients who have been discharged or transferred, or for surgical cases that have been cancelled. ${ }^{31,47,49,50}$

Administration

Prepared drugs are unsupervised and unsecured Prepared drugs left unsecured in clinical areas ${ }^{51}$ are prone to diversion (eg, unlabeled prepared syringes may be replaced with syringes of saline). Drugs can also be withdrawn from active IV infusions (eg, patient-controlled analgesia (PCA) pumps). ${ }^{33,48}$

Unsupervised access to drug stock in patient HCWs may not lock drug inventory (eg, an anesthesiologist may leave the room without locking the drug cart, 52 care areas nurses may forget to log out of the $\left.A D C^{46}\right)$.

ADC may not be optimally configured, updated, $A D C s$ may allow users to perform a "critical override" when the pharmacy is closed, ${ }^{46,53}$ granting access to or monitored drugs normally requiring pharmacy review; if this access is not regularly reviewed the override feature can be abused.

Access privileges to the ADC may not be revoked, providing access to some HCWs longer than appropriate. ${ }^{49}$ ADCs may not automatically log out within a short enough timeframe, falsely tying subsequent withdrawals to the original user. ${ }^{46} \mathrm{ADC}$, if not rapidly updated, can dispense drugs for patients that have already been discharged or transferred. ${ }^{46,47}$

Flexibility in administration

HCWs may be given a high degree of autonomy ${ }^{10}$ and flexibility, which can create opportunities for diversion (eg, flexible ordering [see 'Prescribing']) can increase latitude for unnecessary dispensing, ${ }^{39}$ delays between dispensing and wasting facilitates diversion of partial doses, ${ }^{44}$ sloppy recordkeeping can obscure traceability ${ }^{49,50,54)}$. Intravenous infusions can be prematurely replaced, or fentanyl patches reused, to make additional drug available for diversion. ${ }^{47}$

Falsification of patient documentation HCWs may document complete administration of a drug when some or all of the dose was diverted, $4,22,31,37,39,41,50$ and/or may falsely report pain scores to support apparently higher dose administration. ${ }^{48,55,56}$

Wastage, returns and disposal Visual confirmation of wasting cannot detect Individuals diverting drugs may replace the contents of a syringe with saline before requesting a witness. ${ }^{12,5}$ drug content

\begin{tabular}{|c|c|}
\hline Falsification of drug expiry & $\begin{array}{l}\text { Prematurely expiring valid drugs allows them to be transferred to a separate area; }{ }^{20} \text { these drugs may then be } \\
\text { less frequently audited thereafter, and at higher risk of diversion. }\end{array}$ \\
\hline $\begin{array}{l}\text { Presence of partially administered drugs } \\
\text { on clinical units }\end{array}$ & $\begin{array}{l}\text { Drugs yet to be fully administered (eg, unfinished infusions }{ }^{10} \text { ) may be left unmonitored in clinical areas and } \\
\text { diverted. } .^{19,32,51} \text { Overfill in an injectable vial can be diverted. } .^{58}\end{array}$ \\
\hline Unsecured waste receptacles & Drugs may be removed from sharps receptacles. ${ }^{4,21,34,47,54,59,60}$ Expired drugs may be diverted from holding areas. ${ }^{23,31,35}$ \\
\hline $\begin{array}{l}\text { Complacency in the enforcement } \\
\text { of wasting procedures }\end{array}$ & $\begin{array}{l}\text { Optimal practices may not be regularly reinforced (eg, drugs accidentally taken home in HCWs' pockets, }{ }^{46} \text { lack } \\
\text { of adherence to proper drug wasting procedure }{ }^{12} \text { ). }\end{array}$ \\
\hline Falsification of witnessing & $\begin{array}{l}\text { HCWs may verify wastage without actually witnessing it. } 4,42,50 \mathrm{~A} \text { colleague's credentials can be used to } \\
\text { document that wastage was witnessed, without their presence. }\end{array}$ \\
\hline
\end{tabular}

aFor convenience, storage is placed after the procurement stage of the medication-use process because the largest storehouse of controlled drug likely exists in the hospital pharmacy. However, storage occurs elsewhere (eg, in patient areas, delivery trucks) and readers should be cognizant that storage risks occur at multiple stages of the medication-use process, rather than as a discrete step as it may appear in the table.

Abbreviations: ADC, automated dispensing cabinet; HCW, healthcare worker; PCA, patient-controlled analgesia. 
TABLE 3. Diversion Safeguards at Each Stage of the Medication-Use Process with Associated List of References ${ }^{a}$

\begin{tabular}{lll}
\hline Stage of Medication-Use Process & Safeguard & Description, Examples, and Associated References \\
\hline Procurement & Separate purchasing and receiving roles & $\begin{array}{l}\text { Regularly rotate healthcare workers (HCWs) associated with inventory control roles (eg, purchase, } \\
\text { discrepancy resolution, auditing) }{ }^{35} \text { Provide the minimum information necessary for a purchaser to } \\
\text { generate orders to replenish controlled drugs. }{ }^{35}\end{array}$ \\
\cline { 2 - 3 } & $\begin{array}{l}\text { Periodically audit and reconcile vault inventory } \\
\text { against purchasing and receiving records }\end{array}$ & $\begin{array}{l}\text { Periodically audit inventory, particularly controlled drugs stored in the pharmacy vault. }{ }^{31,35} \text { Reconcile } \\
\text { financial statements and wholesale purchase history with inventory; this may identify cases where the } \\
\text { purchase orders and packing slips (as a pair) have been removed. }{ }^{31,40,61} \text { Maintain a separate log of all } \\
\text { purchase orders so they can be reconciled against the vault records. }{ }^{35} \text { Establish a process to identify } \\
\text { unusual peaks in quantity or frequency of controlled-drug purchases. }{ }^{31}\end{array}$ \\
\end{tabular}

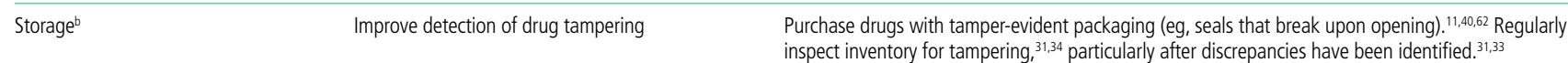

Enable processes in the pharmacy that enforce Establish clear audit trails for all controlled-drug access (ie, who accessed substances and when, what documentation and traceability of controlled-drug changes were made). ${ }^{63}$ Cameras recording critical areas (eg, controlled-drug vault) will help identify who inventory and all who have accessed it has accessed inventory. ${ }^{11,21,35,40,61}$ Blind counts should be used in the pharmacy when accessing controlled drugs; and users should identify how much is to be removed before gaining access..$^{40}$ Establish dedicated human resources to audit access reports and known risk points (eg, repackaged products). ${ }^{35}$ Ensure that expiry dates are accurate in inventory documentation, and eliminate other sources of discrepancies (eg, labeling on products differing from product records in electronic system). ${ }^{64}$ Audit multi-dose or bulk transactions to account for each milligram of drug. ${ }^{35}$

Maximize security of drugs within the pharmacy Limit access to inventory areas to appropriate HCWs (and only on days when they are scheduled to work), and minimize unnecessary traffic (eg, personal belongings never kept in drug storage areas). 10,61 Secure multidose vials when not in use (eg, in a locked refrigerator). ${ }^{35}$ Ensure that key/code access is tightly controlled, and establish a process to update keys/codes regularly. ${ }^{38,40,52}$

\begin{tabular}{|c|c|c|}
\hline \multirow[t]{3}{*}{ Prescribing } & $\begin{array}{l}\text { Establish processes to identify unusual or } \\
\text { inappropriate prescribing }\end{array}$ & $\begin{array}{l}\text { Do not allow prescribers to prescribe drug for themselves or for friends/family. }{ }^{40} \text {, Identify unusual } \\
\text { prescribing trends or patterns (eg, variance compared to peers). }{ }^{31} \text { Audit compliance with verbal order } \\
\text { policy: }{ }_{i}^{65} \text { large numbers of rejected verbal orders may be cause for suspicion. }{ }^{33,47}\end{array}$ \\
\hline & Reduce range orders & $\begin{array}{l}\text { Where feasible, restrict the use of dosing ranges }{ }^{40} \text { this prevents } \mathrm{HCWs} \text { seeking to divert from } \\
\text { preferentially documenting larger doses to facilitate their diversion. Frequent assessments of the patient's } \\
\text { sedation may reduce the amount of drug administered }{ }^{66} \text { and therefore the amount available for diversion. }\end{array}$ \\
\hline & Avoid forged prescriptions & $\begin{array}{l}\text { Ensure that prescription pads/papers are kept secure and are tamper-resistant; ensure that electronic } \\
\text { order entry/e-prescribing systems are secure. }{ }^{21,31,32,35,36,40}\end{array}$ \\
\hline Preparation & Avoid compounding and repackaging & $\begin{array}{l}\text { Purchase unit-dose drugs where possible, to minimize requirements for repackaging of drugs in } \\
\text { pharmacy and thus minimize opportunities for diversion. }{ }^{40}\end{array}$ \\
\hline \multirow[t]{2}{*}{ Dispensing } & Log drug movement into and out of the pharmacy & $\begin{array}{l}\text { Log drugs entering or leaving the pharmacy to support auditing, with identification and resolution of } \\
\text { discrepancies daily, ideally by a HCW who is not routinely involved in handling controlled drugs. }{ }^{35} \text { Include } \\
\text { in the log: dispensation by hand, dispensation to unit automated dispensing cabinets (ADCs), and other } \\
\text { deliveries. }{ }^{40,67} \text { Deliveries to non-ADC areas should be co-signed by the delivery person and the receiver, } \\
\text { and the drugs should be immediately secured on the unit. }\end{array}$ \\
\hline & $\begin{array}{l}\text { Reduce unnecessary supply and access to controlled } \\
\text { drugs on clinical units }\end{array}$ & $\begin{array}{l}\text { Limit quantities of drugs stocked in the unit, and restock frequently; use unit doses where possible, to } \\
\text { reduce drug waste susceptible to diversion. }{ }^{68} \text { Avoid placing controlled drugs in matrix-type drawers that } \\
\text { accommodate multiple products (wherever possible, access should be limited to only the desired drugs). }{ }^{68} \\
\text { When the pharmacy is closed, limit the supply of controlled drugs for urgent orders. For surgical teams, } \\
\text { consider limiting the supply of narcotics (per procedure or daily), to maximize individual accountability } \\
\text { and simplify the audit trail; reconciliation of the administration record with the dispensing record and } \\
\text { wastage should occur immediately to identify and resolve discrepancies. }{ }^{69} \text { Controlled-drug inventory } \\
\text { levels are routinely reviewed, and orders are based on usage to minimize excess stock. }{ }^{31}\end{array}$ \\
\hline
\end{tabular}

Continued on page 425

Hospitals may also consider monitoring whether staff access controlled-drug areas when they are not scheduled to work to detect security breaches.

Safeguards for both issues benefit from an organizational culture reinforced through training at orientation and annually thereafter. Staff should be aware of reporting mechanisms (eg, anonymous hotlines), employee and professional assistance programs, self-reporting protocols, and treatment and rehabilitation options. ${ }^{10,12,29,47,72,91}$ Other system-wide safeguards described in Table 3 should also be considered. Detection of transactional discrepancies does not automatically indicate diversion, but recurrent discrepancies indicate a weakness in controlled-drug management and should be rectified; diversion prevention is a responsibility of all departments, not just the pharmacy.

Hospitals have several motivations to actively invest in safeguards. Drug diversion is a patient safety issue, a patient privacy issue (eg, patient records are inappropriately accessed to identify opportunities for diversion), an occupational health issue given the higher risks of opioid-related SUD faced by HCWs, a regulatory compliance issue, and a legal issue. ${ }^{31,41,46,59,78,98,99}$ Although individuals are accountable for drug diversion itself, hospitals should take adequate measures to prevent or detect diversion and protect patients and staff from associated harms. Hospitals should pay careful attention to the configuration of healthcare technologies, environments, and processes in their institution to reduce the opportunity for diversion.

Our study has several limitations. We did not include articles prior to 2005 because we captured a sizable amount of literature with the current search terms and wanted the majority of the studies to reflect workflow based on electronic 


\section{TABLE 3. Diversion Safeguards at Each Stage of the Medication-Use Process with Associated List of References} (continued)

\begin{tabular}{ll}
\hline Stage of Medication-Use Process & Safeguard \\
\hline Administration & Minimize the use of critical override function \\
& \\
& Ensure clinical documentation is accurate to enable \\
detection of abnormal patterns in drug access
\end{tabular}

\section{Description, Examples, and Associated References}

ADCs may allow users to access specific drugs when the pharmacy is closed or in critical emergent situations (typically via a "critical override"). Eliminate this function, or institute tight control with co-signatures and frequent auditing. ${ }^{40,68}$ Other approaches to override controls (eg, in the case of power outages, system failures) should also be audited and evaluated for security. ${ }^{46,68}$ Avoid the need for nonpharmacists to have access to the pharmacy by properly stocking night cabinets, drug carts or ADCs during pharmacy off-hours. ${ }^{68}$

detection of abnormal patterns in drug access

Ensure records accurately capture which HCWs are accessing what drugs and when to ensure that the amount of drug ordered, administered, and wasted is clinically appropriate. ${ }^{21,56,63,70}$ The number of discrepancies should be tracked, and resolved within 24 hours, and 72 hours at most. 15,31,46 Electronic systems (eg, ADCs) may support accurate documentation and trigger alerts (eg, HCWs withdrawing more drug than their peers will be flagged). ${ }^{71}$ After-hours drug access and repeating pairs of co-signers should also be identified. ${ }^{10,31,43,72}$

Nonclinical systems (eg, key-card access and shift schedules) should also be consulted to identify HCWs: accessing drugs or documentation when not on duty, accessing ADCs outside their work area, or associating with patients who have been affected by outbreaks of blood-borne pathogens (eg, hepatitis ( virus). ${ }^{11,53}$

For patient-controlled analgesia, institute a co-signature process for pump cartridges, keep such cartridges secure in a locked infusion pump; ${ }^{10,21}$ require that a witness observe the waste disposal process once the cartridge is removed from the pump..$^{31}$ Where possible, use portless intravenous infusion tubing for controlled-drug infusions, lock pump interfaces (to limit manipulation of infusion rate and/or volume) and clearly document volumes infused, infusion rate and boluses for reconciliation at shift change. ${ }^{10}$

Minimize credential sharing between HCWs and ensure access privileges are updated frequently

Passwords and/or identification badges should never be shared between $\mathrm{HCWs}^{38,56}$ the use of biometric access may reduce the risk of credential sharing..$^{37,40,61,73,74}$ Ensure that $A D C s$ are updated regularly to capture staffing changes, and changes to patient profiles (eg, discharged patients should not appear in the $A D C$ patient list). ${ }^{46}$

Support drug-handling procedures that promote accountability and security

Maximize the security of dispensed drug: provide containers for carrying drugs to the bedside to minimize risk of being left unattended; 68 such containers help to secure controlled drug before and after administration, before disposal of waste. However, these containers should only be accessible to authorized personnel when not in use. ${ }^{31}$

Limit amount of dispensed drug: limit drug retrieval to the current doses required for a single patient. ${ }^{56}$ Prohibit withdrawal of more than a single dose of a controlled drug into a syringe, so that partial doses are not vulnerable to diversion. ${ }^{21}$

Maximize accountability for dispensed drug: the HCW retrieving the drug should be the person who administers, to optimize accountability. ${ }^{33,40}$ Assigning patients to specific HCWs may increase accountability and traceability of drug administration. ${ }^{33,55}$

Reduce opportunities for diversion between drug withdrawal and administration

Define a specific interval within which drugs should be administered after retrieval. ${ }^{40}$ Ensure that the number, size and location of ADCs is appropriate for the clinical unit (ie, no more than $30 \mathrm{~m}$ from patients' rooms) to support usability, efficiency and compliance. ${ }^{75} \mathrm{HCW}$ should use ADCs only in their primary work area. Account for nurses' requirements and concerns, which may differ from those of the pharmacy (eg, nursing workflow may require more counter space and multi-tasking across multiple medical devices than pharmacists would consider). ${ }^{75}$ Label any syringes containing drugs that are not administered immediately, in accordance with institutional policy. 10,40,52,62

Wastage, returns, and disposal Audit wasted drugs using assay technologies Consider random assays of drugs returned to the pharmacy via refractometry or ultraviolet spectroscopy, with recognition that each approach has its limitations (eg, accuracy and cost). 4,31,35,49,69,7,7,77

Reinforce the need for appropriate witnessing Establish processes to ensure that all waste is witnessed in real time with visual line of sight; witnessing after the fact is unacceptable. ${ }^{21,56}$ Some guidelines suggest wasting occur at time of withdrawal from secure storage. ${ }^{78}$ The transfer of controlled drugs to a destruction company should also be witnessed and co-signed. .5

Secure wasted and expired drugs

Frequently remove expired items to prevent accumulation of drug. 21,23,35 Sharps/waste receptacles should prevent drugs and waste from being shaken out or attempts to forcibly reach into openings. ${ }^{21}$ If larger containers must be used, video cameras may be helpful to monitor their status. ${ }^{21}$ Lock waste receptacles to the wall or other stationary equipment so that they cannot be easily removed from a clinical unit; keys allowing replacement of containers should be limited to a few designated HCWs. ${ }^{21}$

Audit and reconcile documentation to verify wastage Verify and audit the return of drugs intended for disposal to the pharmacy (eg, require co-signatures from the responsible HCW and the recipient in the pharmacy). All other wastage should be witnessed and co-signed. ${ }^{21,31,56}$ Where possible, correlate quantities administered with quantities dispensed (eg, dispensations per surgical case). Reconcile the list of controlled drugs sent for disposal against reports from the destruction company, to ensure that all items are accounted for. ${ }^{4,65}$ 
TABLE 3. Diversion Safeguards at Each Stage of the Medication-Use Process with Associated List of References ${ }^{2}$ (continued)

\begin{tabular}{|c|c|c|}
\hline Stage of Medication-Use Process & Safeguard & Description, Examples, and Associated References \\
\hline System-wide ${ }^{c}$ & Pre-employment screening & $\begin{array}{l}\text { Reference and background checks should be conducted } 10,12,18,60,62,70 \text { to prevent hiring HCWs with known } \\
\text { or suspected drug diversion histories. }{ }^{17,51} \text { High-risk roles (eg, pharmacy staff), should be rescreened on } \\
\text { an annual basis after hire. }{ }^{20} \text { Consider existing regulations where available. For example, the US Drug } \\
\text { Enforcement Agency (DEA) suggests specific questions regarding the candidate's criminal history during } \\
\text { pre-employment screens. } .^{10,60} \text { Question references with knowledge of candidate's clinical behavior rather } \\
\text { than purely administrative details. }{ }^{10} \text { Note, employers can be reluctant to disclose poor job performance } \\
\text { due to liability risks. } .^{19,51,53} \text { Reforms have been suggested to improve disclosure from employers. } .^{51,79}\end{array}$ \\
\hline
\end{tabular}

Implement a drug testing program Drug testing of HCWs has been used in three capacities: pre-employment screening, random testing on an ongoing basis, and when there is reason to suspect impairment (ie, "for cause" drug testing). ${ }^{18,20,27,60,80-89}$ Some authors caution against random drug testing..$^{86,87,90}$ Drug testing is connected with issues of legal discovery and confidentiality, which vary according to local and federal regulations; review the provided references and refer to local statutes and counsel.

Educate staff

Educating HCWs is essential to diversion prevention; 4,10,12,31,91 preferably during orientation and annually thereafter. ${ }^{4,38,74}$ Key concepts include: raising awareness that diversion occurs in all facilities where controlled drugs are handled, ${ }^{12}$ identifying drugs that are prone to diversion, describing characteristics of individuals seeking to divert drugs, ensuring that drug-handling policies and procedures are understood, explaining the importance of reporting and how to do so, and deterring diversion by explaining detection practices that are in place. ${ }^{42,43}$ Random in-person audits of clinical units (eg, compliance checks) can assess staff awareness of preferred practices and reinforce adherence. ${ }^{32,60}$

Establish dedicated diversion investigation teams

Commit staff resources to prevent, detect and respond to diversion. ${ }^{4,10,31,44,46-49,56,74}$ Staff may monitor data from a variety of sources: ADCs and associated analytics, ${ }^{10,12,21,37,57,67-69,71,73,78,92,93}$ video surveillance ${ }^{11,21}$ (while being mindful of patient privacy ${ }^{94}$ ), drug assaying technologies (to verify the contents of wasted drugs), 4,35,49,69,76,77 and biometric access. ${ }^{37,40,61,73,74}$ Diversion teams audit high-risk drugs (eg, narcotics, benzodiazepines, propofol, gabapentin) and follow-up on cues that may indicate potential diversion, such as reports of HCW behaviors, ${ }_{10}^{10,12,22,34,56,79,81}$ patients' reports of untreated pain, ${ }^{10,31}$ or outbreaks where multiple patients are infected with the exact same strain (an outcome consistent with a single infectious HCW who may be self-injecting in the course of their diversion). ${ }^{11,52,62}$ These teams can use a standard investigation protocol to ensure HCW confidentiality, equal treatment of all employees, optimize evidence-handling procedures, and minimize legal criticisms of how investigations were handled. .00,92,95 $^{2}$

aTable 3 is not intended to provide a direct one-to-one mapping with the contributors to diversion described in Table 2

bFor convenience, storage is placed after the procurement stage of the medication-use process because the largest storehouse of controlled drug likely exists in the hospital pharmacy. However, storage occurs elsewhere (eg, in patient areas, delivery trucks) and readers should be cognizant that storage risks occur at multiple stages of the medication-use process, rather than as a discrete step as it may appear in the table.

cSystem-wide refers to safeguards that apply to multiple stages of the medication-use process

Abbreviations: ADC, automated dispensing cabinet; DEA, drug enforcement agency; $\mathrm{HCW}$, healthcare worker.

health records and medication ordering, which only came into wide use in the past 15 years. Other possible contributors and safeguards against drug diversion may not be captured in our review. Nevertheless, thorough consideration of the two underlying issues described will help protect hospitals against new and emerging methods of diversion. The literature search yielded a paucity of controlled trials formally evaluating the effectiveness of these interventions, so safeguards identified in our review may not represent optimal strategies for responding to drug diversion. Lastly, not all suggestions may be applicable or effective in every institution.

\section{CONCLUSION}

Drug diversion in hospitals is a serious and urgent concern that requires immediate attention to mitigate harms. Past incidents of diversion have shown that hospitals have not yet implemented safeguards to fully account for drug losses, with resultant harms to patients, HCWs, hospitals themselves, and the general public. Further research is needed to identify system factors relevant to drug diversion, identify new safeguards, evaluate the effectiveness of known safeguards, and support adoption of best practices by hospitals and regulatory bodies.

\section{Acknowledgments}

The authors wish to thank Iveta Lewis and members of the HumanEra team (Carly Warren, Jessica Tomasi, Devika Jain, Maaike deVries, and Betty Chang) for screening and data extraction of the literature and to Peggy Robinson, Sylvia Hyland, and Sonia Pinkney for editing and commentary.

Disclosures: Ms. Reding and Ms. Hyland were employees of North York General Hospital at the time of this work. Dr. Hamilton and Ms. Tscheng are employees of ISMP Canada, a subcontractor to NYGH, during the conduct of the study. Mark Fan and Patricia Trbovich have received honoraria from BD Canada for presenting preliminary study findings at $\mathrm{BD}$ sponsored events.

Funding: This work was supported by Becton Dickinson (BD) Canada Inc. (grant \#ROR2017-04260JH-NYGH). BD Canada had no involvement in study design; in the collection, analysis or interpretation of data; in the writing of the report; or in the decision to submit the article for publication.

\section{References}

1. International Narcotics Control Board. Narcotic drugs: estimated world requirements for 2017 - statistics for 2015. https://www.incb.org/documents/ Narcotic-Drugs/Technical-Publications/2016/Narcotic_Drugs_Publication_2016.pdf. Accessed February 2, 2018.

2. Gomes T, Tadrous M, Mamdani MM, Paterson JM, Juurlink DN. The burden of opioid-related mortality in the United States. JAMA Netw Open. 2018;1(2):e180217. doi: 10.1001/jamanetworkopen.2018.0217.

3. Special Advisory Committee on the Epidemic of Opioid Overdoses. National report: apparent opioid-related deaths in Canada (December 2017). https://www.canada.ca/en/public-health/services/publications/healthy-liv- 
ing/apparent-opioid-related-deaths-report-2016-2017-december.html. Accessed June 5, 2018.

4. Berge KH, Dillon KR, Sikkink KM, Taylor TK, Lanier WL. Diversion of drugs within health care facilities, a multiple-victim crime: patterns of diversion, scope, consequences, detection, and prevention. Mayo Clin Proc 2012;87(7):674-682. doi: 10.1016/j.mayocp.2012.03.013.

5. Inciardi JA, Surratt HL, Kurtz SP, Burke JJ. The diversion of prescription drugs by health care workers in Cincinnati, Ohio. Subst Use Misuse. 2006;41(2):255264. doi: 10.1080/10826080500391829.

6. Hulme S, Bright D, Nielsen S. The source and diversion of pharmaceutical drugs for non-medical use: A systematic review and meta-analysis. Drug Alcohol Depend. 2018;186:242-256. doi: 10.1016/j.drugalcdep.2018.02.010.

7. Minnesota Hospital Association. Minnesota controlled substance diversion prevention coalition: final report. https://www.mnhospitals.org/Portals/0/ Documents/ptsafety/diversion/drug-diversion-final-report-March2012.pdf. Accessed July 21, 2017

8. Joranson DE, Gilson AM. Drug crime is a source of abused pain medications in the United States. J Pain Symptom Manag. 2005;30(4):299-301. doi: 10.1016/j.jpainsymman.2005.09.001.

9. Carman T. Analysis of Health Canada missing controlled substances and precursors data (2017). Github. https://github.com/taracarman/drug_losses. Accessed July 1, 2018.

10. New K. Preventing, detecting, and investigating drug diversion in health care facilities. Mo State Board Nurs Newsl. 2014;5(4):11-14.

11. Schuppener LM, Pop-Vicas AE, Brooks EG, et al. Serratia marcescens Bacteremia: Nosocomial Clustercluster following narcotic diversion. Infect Control Hosp Epidemiol. 2017;38(9):1027-1031. doi: 10.1017/ice.2017.137

12. New K. Investigating institutional drug diversion. J Leg Nurse Consult 2015;26(4):15-18. doi: https://doi.org/10.1016/S2155-8256(15)30095-8

13. Berge $\mathrm{KH}$, Lanier WL. Bloodstream infection outbreaks related to opioid-diverting health care workers: a cost-benefit analysis of prevention and detection programs. Mayo Clin Proc. 2014;89(7):866-868. doi: 10.1016/j. mayocp.2014.04.010.

14. Horvath C. Implementation of a new method to track propofol in an endoscopy unit. Int J Evid Based Healthc. 2017;15(3):102-110. doi: 10.1097/ XEB.0000000000000112.

15. Pontore KM. The Epidemic of Controlled Substance Diversion Related to Healthcare Professionals. Graduate School of Public Health, University of Pittsburgh; 2015.

16. Knowles M. Georgia health system to pay $\$ 4.1 \mathrm{M}$ settlement over thousands of unaccounted opioids. Becker's Hospital Review. https://www.beckershospitalreview.com/opioids/georgia-health-system-to-pay-4-1m-settlement-overthousands-of-unaccounted-opioids.html. Accessed September 11, 2018.

17. Olinger D, Osher CN. Drug-addicted, dangerous and licensed for the operating room. The Denver Post. https://www.denverpost.com/2016/04/23/ drug-addicted-dangerous-and-licensed-for-the-operating-room. Accessed August 2, 2017

18. Levinson DR, Broadhurst ET. Why aren't doctors drug tested? The New York Times. https://www.nytimes.com/2014/03/13/opinion/why-arent-doctorsdrug-tested.html. Accessed July 21, 2017.

19. Eichenwald K. When Drug Addicts Work in Hospitals, No One is Safe. News week. https://www.newsweek.com/2015/06/26/traveler-one-junkies-harrowing-journey-across-america-344125.html. Accessed August 2, 2017.

20. Martin ES, Dzierba SH, Jones DM. Preventing large-scale controlled substance diversion from within the pharmacy. Hosp Pharm. 2013;48(5):406-412 doi: 10.1310/hpj4805-406.

21. Institute for Safe Medication Practices. Partially filled vials and syringes in sharps containers are a key source of drugs for diversion. Medication safety alerts. https://www.ismp.org/resources/partially-filled-vials-and-syringes-sharps-containers-are-key-source-drugs-diversion?id=1132. Accessed June 29, 2017.

22. Fleming K, Boyle D, Lent WJB, Carpenter J, Linck C. A novel approach to monitoring the diversion of controlled substances: the role of the pharmacy compliance officer. Hosp Pharm. 2007;42(3):200-209. doi: 10.1310/hpj4203-200.

23. Merlo LJ, Cummings SM, Cottler LB. Prescription drug diversion among substance-impaired pharmacists. Am J Addict. 2014;23(2):123-128. doi: 10.1111/j.1521-0391.2013.12078.x

24. O'Malley L, Croucher K. Housing and dementia care-a scoping review of the literature. Health Soc Care Commun. 2005;13(6):570-577. doi: 10.1111/j.13652524.2005.00588.x

25. Fischer B, Bibby M, Bouchard M. The global diversion of pharmaceutical drugs non-medical use and diversion of psychotropic prescription drugs in North America: a review of sourcing routes and control measures. Addiction. 2010;105(12):2062-2070. doi: 10.1111/j.1360-0443.2010.03092.x.

26. Inciardi JA, Surratt HL, Cicero TJ, et al. The "black box" of prescription drug diversion. J Addict Dis. 2009;28(4):332-347. doi: 10.1080/10550880903182986.

27. Boulis S, Khanduja PK, Downey K, Friedman Z. Substance abuse: a national survey of Canadian residency program directors and site chiefs at university-affiliated anesthesia departments. Can J Anesth. 2015;62(9):964-971. doi: 10.1007/s12630-015-0404-1.

28. Warner DO, Berge K, Sun H et al. Substance use disorder among anesthesiology residents, 1975-2009. JAMA. 2013;310(21):2289-2296. doi: 10.1001/ jama.2013.281954.

29. Kunyk D. Substance use disorders among registered nurses: prevalence, risks and perceptions in a disciplinary jurisdiction. J Nurs Manag. 2015;23(1):54-64. doi: 10.1111/jonm.12081.

30. Arksey H, O'Malley L. Scoping studies: towards a methodological framework. Int J Soc Res Methodol. 2005;8(1):19-32. doi: 10.1080/1364557032000119616.

31. Brummond PW, Chen DF, Churchill WW, et al. ASHP guidelines on preventing diversion of controlled substances. Am J Health System Pharm. 2017;74(5):325-348. doi: 10.2146/ajhp160919.

32. New K. Diversion risk rounds: a reality check on your drug-handling policies. http://www.diversionspecialists.com/wp-content/uploads/Diversion-RiskRounds-A-Reality-Check-on-Your-Drug-Handling-Policies.pdf. Accessed July 13, 2017.

33. New K. Uncovering Diversion: 6 case studies on Diversion. https://www. pppmag.com/article/2162. Accessed January 30, 2018

34. New KS. Undertaking a System Wide Diversion Risk Assessment [PowerPoint]. International Health Facility Diversion Association Conference; 2016. Accessed July 14, 2016.

35. O'Neal B, Siegel J. Diversion in the pharmacy. Hosp Pharm. 2007;42(2):145148. doi: 10.1310/hpj4202-145

36. Holleran RS. What is wrong With this picture? J Emerg Nurs. 2010;36(5):396397. doi: 10.1016/j.jen.2010.08.005.

37. Vrabel R. Identifying and dealing with drug diversion. How hospitals can stay one step ahead. https://www.hcinnovationgropu.com/home/article/13003330/identifying-and-dealing-with-drug-diversion. Accessed September 18, 2017

38. Mentler P. Preventing diversion in the ED. https://www.pppmag.com/article/1778. Accessed July 19, 2017.

39. Fernandez J. Hospitals wage battle against drug diversion. https://www. drugtopics.com/top-news/hospitals-wage-battle-against-drug-diversion. Accessed August 17, 2017

40. Minnesota Hospital Association. Road map to controlled substance diversion Prevention 2.0. https://www.mnhospitals.org/Portals/O/Documents/ ptsafety/diversion/Road Map to Controlled Substance Diversion Prevention 2.0.pdf. Accessed June 30, 2017

41. Bryson EO, Silverstein JH. Addiction and substance abuse in anesthesiology. Anesthesiology. 2008;109(5):905-917. doi: 10.1097/ALN.0b013e3181895bc1.

42. McCammon C. Diversion: a quiet threat in the healthcare setting. https:// www.acep.org/Content.aspx?ID=94932. Accessed August 17, 2017.

43. Minnesota Hospital Association. Identifying potentially impaired practitioners [PowerPoint]. https://www.mnhospitals.org/Portals/0/Documents/ptsafety/ diversion/potentially-impaired-practitioners.pdf. Accessed July 21, 2017.

44. Burger G, Burger M. Drug diversion: new approaches to an old problem. Am J Pharm Benefits. 2016;8(1):30-33.

45. Greenall J, Santora P, Koczmara C, Hyland S. Enhancing safe medication use for pediatric patients in the emergency department. Can J Hosp Pharm. 2009;62(2):150-153. doi: 10.4212/cjhp.v62i2.445.

46. New K. Avoid diversion practices that prompt DEA investigations. https:// www.pppmag.com/article/1818. Accessed October 4, 2017.

47. New K. Detecting and responding to drug diversion. https://rxdiversion. com/detecting-and-responding-to-drug-diversion. Accessed July 13, 2017.

48. New KS. Institutional Diversion Prevention, Detection and Response [PowerPoint]. https://www.ncsbn.org/0613_DISC_Kim_New.pdf. Accessed August 25, 2017.

49. Siegel J, Forrey RA. Four case studies on diversion prevention. https://www. pppmag.com/article/1469/March_2014/Four_Case_Studies_on_Diversion_ Prevention. Accessed July 31, 2017.

50. Copp MAB. Drug addiction among nurses: confronting a quiet epidemic-Many RNs fall prey to this hidden, potentially deadly disease. http://www. modernmedicine.com/modern-medicine/news/modernmedicine/modern-medicine-feature-articles/drug-addiction-among-nurses-con. Accessed September 8, 2017.

51. Maryland Department of Health and Mental Hygiene. Public health vulnerability review: drug diversion, infection risk, and David Kwiatkowski's employment as a healthcare worker in Maryland. https://health.maryland.gov/pdf/ Public Health Vulnerability Review.pdf. Accessed July 21, 2017.

52. Warner AE, Schaefer MK, Patel PR, et al. Outbreak of hepatitis $C$ virus in- 
fection associated with narcotics diversion by an hepatitis $C$ virus-infected surgical technician. Am J Infect Control. 2015;43(1):53-58. doi: 10.1016/j. ajic.2014.09.012.

53. New Hampshire Department of Health and Human Services-Division of Public Health Services. Hepatitis C outbreak investigation Exeter Hospital public report. https://www.dhhs.nh.gov/dphs/cdcs/hepatitisc/documents/hepcoutbreak-rpt.pdf. Accessed July 21, 2017

54. Paparella SF. A tale of waste and loss: lessons learned. J Emerg Nurs. 2016;42(4):352-354. doi: 10.1016/j.jen.2016.03.025

55. Ramer LM. Using servant leadership to facilitate healing after a drug diversion experience. AORN J. 2008;88(2):253-258. doi: 10.1016/j.aorn.2008.05.002.

56. Siegel J, O'Neal B, Code N. Prevention of controlled substance diversion-Code N: multidisciplinary approach to proactive drug diversion prevention. Hosp Pharm. 2007;42(3):244-248. doi: 10.1310/hpj4203-244.

57. Saver C. Drug diversion in the OR: how can you keep it from happen ing? https://pdfs.semanticscholar.org/f066/32113de065ca628a1f37218d18c654c15671.pdf. Accessed September 21, 2017

58. Peterson DM. New DEA rules expand options for controlled substance disposal. J Pain Palliat Care Pharmacother. 2015;29(1):22-26. doi: 10.3109/15360288.2014.1002964.

59. Lefebvre LG, Kaufmann IM. The identification and management of substance use disorders in anesthesiologists. Can J Anesth J Can Anesth 2017;64(2):211-218. doi: 10.1007/s12630-016-0775-y.

60. Missouri Bureau of Narcotics \& Dangerous Drugs. Drug diversion in hospitals-A guide to preventing and investigating diversion issues. https://health. mo.gov/safety/bndd/doc/drugdiversion.doc. Accessed July 21, 2017

61. Hayes S. Pharmacy diversion: prevention, detection and monitoring: a pharmacy fraud investigator's perspective. International Health Facility Diversion Association Conference 2016. Accessed July 5, 2017.

62. Schaefer MK, Perz JF. Outbreaks of infections associated with drug diversion by US health care personnel. Mayo Clin Proc. 2014;89(7):878-887. doi: 10.1016/j.mayocp.2014.04.007

63. Vigoda MM, Gencorelli FJ, Lubarsky DA. Discrepancies in medication entries between anesthetic and pharmacy records using electronic databases. Anesth Analg. 2007;105(4):1061-1065. doi: 10.1213/01.ane.0000282021.74832.5e

64. Goodine C. Safety audit of automated dispensing cabinets. https://www.ncbi. nlm.nih.gov/pmc/articles/PMC2832564/. Accessed September 25, 2017.

65. Ontario College of Pharmacists. Hospital assessment criteria. http://www. ocpinfo.com/library/practice-related/download/library/practice-related/ download/Hospital-Assessment-Criteria.pdf. Accessed August 30, 2017

66. Lizza BD, Jagow B, Hensler D, et al. Impact of multiple daily clinical pharmacist-enforced assessments on time in target sedation range. J Pharm Pract. 2018;31(5):445-449. doi: 10.1177/0897190017729522

67. Landro L. Hospitals address a drug problem: software and Robosts help secure and monitor medications. The Wall Street Journal. https://www.wsj. com/articles/hospitals-address-a-drug-problem-1392762765. Accessed June 29, 2017

68. Hyland S, Koczmara C, Salsman B, Musing ELS, Greenall J. Optimizing the use of automated dispensing cabinets. Can J Hosp Pharm. 2007;60(5):332 334. doi: http://dx.doi.org/10.4212/cjhp.v60i5.205

69. O'Neal B, Bass K, Siegel J. Diversion in the operating room. Hosp Pharm 2007:42(4):359-363. doi: 10.1310/hpj4204-359.

70. White C, Malida J. Large pill theft shows challenge of securing hospita drugs. https://www.matrixhomecare.com/downloads/HRM060110.pdf. Accessed August 18, 2017.

71. Crowson K, Monk-Tutor M. Use of automated controlled substance cabinets for detection of diversion in US hospitals: a national study. Hosp Pharm. 2005;40(11):977-983. doi: 10.1177/001857870504001107.

72. National Council of State Boards of Nursing Inc. Substance use disorder in the workplace [chapter 6]. In: Substance Use Disorder in Nursing. Chicago: National Council of State Boards of Nursing Inc. https://www.ncsbn.org/ SUDN_11.pdf. Accessed. July 21, 2017.

73. Swanson B-M. Preventing prescription drug diversions at your hospital. Campus Safety. http://www.campussafetymagazine.com/cs/preventing-prescription-drug-diversions-at-your-hospital. Accessed June 30, 2017

74. O'Neal B, Siegel J. Prevention of controlled substance diversion-scope, strategy, and tactics: Code $\mathrm{N}$ : the intervention process. Hosp Pharm. 2007:42(7):633-656. doi: 10.1310/hpj4207-653

75. Mandrack M, Cohen MR, Featherling J, et al. Nursing best practices using automated dispensing cabinets: nurses' key role in improving medication safety. Medsurg Nurs. 2012;21(3):134-139.
76. Berge KH, Seppala MD, Lanier WL. The anesthesiology community's approach to opioid- and anesthetic-abusing personnel: time to change course. Anesthesiology. 2008;109(5):762-764. doi: 10.1097/ALN.0b013e31818a3814.

77. Gemensky J. The pharmacist's role in surgery: the indispensable asset. US Pharm. 2015;40(3):HS8-HS12.

78. New K. Drug diversion: regulatory requirements and best practices. http:// www.hospitalsafetycenter.com/content/328646/topic/ws_hsc_hsc.html. Accessed September 21, 2017

79. Lahey T, Nelson WA. A proposed nationwide reporting system to satisfy the ethical obligation to prevent drug diversion-related transmission of hepatitis C in healthcare facilities. Clin Infect Dis. 2015;60(12):1816-1820. doi: 10.1093/ $\mathrm{cid} / \mathrm{civ} 203$

80. Gavin KG. Would not-for-cause randomized drug testing reduce the incidence of drug misuse Among certified registered nurse anesthetists? https:// aquila.usm.edu/cgi/viewcontent.cgi?article=1019\&context=dnp_capstone. Accessed July 20, 2017.

81. Tetzlaff J, Collins GB, Brown DL, et al. A strategy to prevent substance abuse in an academic anesthesiology department. J Clin Anesth. 2010;22(2):143150. doi: 10.1016/j.jclinane.2008.12.030.

82. Kintz P, Villain M, Dumestre V, Cirimele V. Evidence of addiction by anesthesiologists as documented by hair analysis. Forensic Sci Int. 2005;153(1):81-84. doi: 10.1016/j.forsciint.2005.04.033.

83. Wolf CE, Poklis A. A rapid HPLC procedure for analysis of analgesic pharmaceutical mixtures for quality assurance and drug diversion testing. J Anal Toxicol. 2005;29(7):711-714. doi: 10.1093/jat/29.7.711.

84. Poklis JL, Mohs AJ, Wolf CE, Poklis A, Peace MR. Identification of drugs in parenteral pharmaceutical preparations from a quality assurance and a diversion program by direct analysis in real-time AccuTOF(TM)-mass spectrometry (Dart-MS). J Anal Toxicol. 2016;40(8):608-616. doi: 10.1093/jat/bkw065

85. Pham JC, Pronovost PJ, Skipper GE. Identification of physician impairment. JAMA. 2013;309(20):2101-2102. doi: 10.1001/jama.2013.4635.

86. Stolbach A, Nelson LS, Hoffman RS. Protection of patients from physician substance misuse. JAMA. 2013;310(13):1402-1403. doi: 10.1001/jama.2013.277948.

87. Berge KH, McGlinch BP. The law of unintended consequences can never be repealed: the hazards of random urine drug screening of anesthesia providers. Anesth Analg. 2017;124(5):1397-1399. doi: 10.1213/ANE.0000000000001972.

88. Oreskovich MR, Caldeiro RM. Anesthesiologists recovering from chemical dependency: can they safely return to the operating room? Mayo Clin Proc. 2009:84(7):576-580. doi: 10.1016/S0025-6196(11)60745-3.

89. Di Costanzo M. Road to recovery. http://rnao.ca/sites/rnao-ca/files-RNJ-JanFeb2015.pdf. Accessed September 28, 2017

90. Selzer J. Protection of patients from physician substance misuse. JAMA. 2013;310(13):1402-1403. doi: 10.1001/jama.2013.277948.

91. Wright RL. Drug diversion in nursing practice a call for professional accountability to recognize and respond. J Assoc Occup Health Prof Healthc. 2013;33(1):27-30

92. Siegel J, O’Neal B, Wierwille C. The investigative process. Hosp Pharm. 2007;42(5):466-469. doi: 10.1310/hpj4205-466.

93. Brenn BR, Kim MA, Hilmas E. Development of a computerized monitoring program to identify narcotic diversion in a pediatric anesthesia practice. Am J Health System Pharm. 2015;72(16):1365-1372. doi: 10.2146/ajhp140691.

94. Drug diversion sting goes wrong and privacy is questioned. http://www.reliasmedia.com/articles/138142-drug-diversion-sting-goes-wrong-and-privacy-is-questioned. Accessed September 21, 2017.

95. New K. Drug diversion defined: steps to prevent, detect, and respond to drug diversion in facilities. CDC's healthcare blog. https://blogs.cdc.gov/ safehealthcare/drug-diversion-defined-steps-to-prevent-detect-and-respond-to-drug-diversion-in-facilities. Accessed July 21, 2017.

96. Howorun C. 'Unexplained losses' of opioids on the rise in Canadian hospitals. Maclean's. http://www.macleans.ca/society/health/unexplained-losses-of-opioids-on-the-rise-in-canadian-hospitals. Accessed December 5, 2017

97. Carman T. When prescription opioids run out, users look for the supply on the streets. CBC News. https://www.cbc.ca/news/canada/when-prescription-opioids-run-out-users-look-for-the-supply-on-the-streets-1.4720952. Accessed July 1, 2018

98. Tanga HY. Nurse drug diversion and nursing leader's responsibilities: legal regulatory, ethical, humanistic, and practical considerations. JONAs Healthc Law Eth Regul. 2011:13(1):13-16. doi: 10.1097/NHL.0b013e31820bd9e6.

99. Scholze AR, Martins JT, Galdino MJQ, Ribeiro RP. Occupational environment and psychoactive substance consumption among nurses. Acta Paul Enferm. 2017;30(4):404-411. doi: 10.1590/1982-0194201700060. 